\title{
OPTIMAL SHAPE OF TRUSS STRUCTURES SUPPORTING PROTECTIVE WALLS
}

\author{
PETR PROCHAZKA \& MARTIN VALEK \\ Civil Engineering, Czech Technical University in Prague, Czech Republic
}

\begin{abstract}
In this paper, design of trusses supporting a protective wall is discussed. The wall is assumed as sufficiently bearable and fully distributes the load caused by explosion. The problem of the assessment is narrowed to the optimal design for minimum overall mass of the trusses. The reason why the problem of such a protective system is often focused on the optimal shape of trusses is the fact that their members may be considered as a system of springs, which are a suitable means of dissipating energy induced by the effect of load due to explosion. The explosion is initiated in space or directly on the surface of the wall. In our case, vertical walls are considered in 2D; simple generalization of the proposed procedure described below can lead to the optimization of trusses that are curved both in the plane and in space. The algorithms put forward can be applied well for these generalized cases of geometry of the trusses. For various load scenarios, depending on the center of explosion, the optimal shape of truss columns are found using a nonlinear programming, based on the extended Simplex method. An important circumstance is the introduction of buckling effect in the compressed members. The joints are fixed in the plane of the truss and the joint connections are selected in such a way that the minimum mass of the whole truss is attained. The approach leading to the optimal shape is briefly described. It is taken into account that the optimal structure of the truss is achieved exclusively for a statically determinate structure, which always exhibits better results than the indeterminate one. Several typical examples accompany the proposed theory.
\end{abstract}

Keywords: protective walls, reinforcing trusses, overall mass optimization, nonlinear programming.

\section{INTRODUCTION}

Optimal mass of selected truss structures is sought, adapting nonlinear programming based on extended Simplex method. In comparison to other publications on this topic, the buckling of compressed members is also involved in the study. A general procedure leading to the optimal arrangement of the members is described and applied to specific cases of truss geometry and the position of the charge.

Always in 1964, [1], it was proved that when considering statically indeterminate truss, then from such a structure one can find certain statically determined truss structure, the induced stresses in all members are equal to the given strength and in the same time such a structure attains the minimum overall mass. This is a very important prerequisite for further considerations in this paper.

The effect of the dynamic load induced by the shock wave on the protective walls is reported in [2], [3]. In [2], certain experiments are carried out and the results are compared with the numerical treatment suggested in the paper; in [3], the effect of a sudden change of temperature induced by the explosion is also considered as an additional load of the structure.

Papers [4], [5], are focused on the effect of explosive load of structures, assuming the center of charge outside the structure. In paper [4], simple experiments are performed, the empirical formulas from which are deduced. In [5], the complex response of elastic laminated archers loaded by the shock wave is studied. In [6], an explosion is initiated in a closed space. Again, both the effect of shock wave and temperature are taking into account. For numerical evaluation of the behavior of the underground structure that interacts with 
the shock wave effect, a new numerical method, free hexagons, is used in [7]. In [8], the evaluation of the response to the explosion load is presented for various shapes of canopied walls, using both the numerical and experimental studies.

Papers [9]-[14] also focus on optimizing truss structures; mainly shapes of trusses are optimized. In [9], various shapes of roof trusses are compared and optimized: pitched, fan, low profile, vault, Polynesian and others. Selected simplified algorithms are applied to solve the problem. In [10], modified teaching - learning based optimization is presented, while in [11], the charged system search algorithm and its enhanced version are used for optimizing various truss structures with multiple frequency constraints. Interactive truss design using Particle Swarm Optimization and NURBS curves is applied in [12]. In the publication, [13], simulated annealing, and in [14], hybrid genetic algorithms, are used to optimize trusses.

Since matrix and vector notations are often used in this paper, it is worth noting that [.] denotes generally rectangular matrix and $\{$.$\} is a vector.$

\section{PRELIMINARY CONSIDERATIONS}

If the truss structure has $J$ nodes, number $N$ of potential members evidently is

$$
N=\frac{J(J-1)}{2}
$$

The member $e$ connecting the nodes $j$ and $k$ is numbered as

$$
e=J(j-1)-\frac{j(j+1)}{2}+k
$$

providing $j<k<J$, and if $k<j<J$ then

$$
e=J(k-1)-\frac{k(k+1)}{2}+j
$$

The forces in the members can either be tensile (positive sign) or compressed (negative sign). This is why, similar to the Simplex method, all variables (forces) admit only nonnegative values; it is formally assumed that twice the number of eligible independent variables in the simplex is considered. For the member $e$, the tension force and the admissible stress in tension (tensile strength) are denoted as $p_{2 e-1}>0$ and $\sigma_{2 e-1}>0$, respectively, and, similarly, the compressive force and the admissible stress in compression (compressive strength) are $p_{2 e}>0$ and $\sigma_{2 e}>0$, respectively. It follows from this notation that the odd subscripts indicate tension and even one compression. In this section, let us omit buckling. Paper [1] points out that from all truss connections, there exists certain statically determined optimal structure which provides minimum overall mass and the stresses in all members are equal to the given strength. No combination of members in a statically in determined truss structure can provide a better solution. Then, the crosssectional areas $A_{2 e}$ and $A_{2 e-1}$ of the member $e$ is derived from the strength in an individual member $e$, 


$$
A_{2 e}=\frac{p_{2 e}}{\sigma_{2 e}} \text { and } A_{2 e-1}=\frac{p_{2 e-1}}{\sigma_{2 e-1}}
$$

In an individual member, always at least one summand disappears. The total mass $M$ of the structure can then be expressed as,

$$
M=\sum_{e=1}^{N}\left\{\frac{p_{2 e-1}}{\sigma_{2 e-1}}+\frac{p_{2 e}}{\sigma_{2 e}}\right\} \rho_{e} l_{e},
$$

where $\rho_{e}$ is the mass density and $l_{e}$ is the length of the member $e$. Introducing

$$
\{C\}=\left\{\frac{\rho_{1} l_{1}}{\sigma_{1}}, \frac{\rho_{1} l_{1}}{\sigma_{2}}, \cdots, \frac{\rho_{e} l_{e}}{\sigma_{2 e-1}}, \frac{\rho_{e} l_{e}}{\sigma_{2 e}}, \cdots, \frac{\rho_{N} l_{N}}{\sigma_{2 N-1}}, \frac{\rho_{N} l_{N}}{\sigma_{2 N}}\right\}^{\mathrm{T}},
$$

and

$$
\{P\}=\left\{p_{1}, p_{2}, \cdots, p_{2 e-1}, p_{2 e}, \cdots, p_{2 N-1}, p_{2 N}\right\}^{\mathrm{T}},
$$

the overall mass equals

$$
M=\{C\}^{\mathrm{T}} \cdot\{P\}
$$

Directional cosines with respect to the coordinate axes $x_{1}$, positive from left to right, of the member $e$ are indicated by $r_{2 e-1}$ and $r_{2 e}$. The coordinate $x_{2}$ is taken positive upwards. These cosines respect the direction from $j$ to $k, j<k$. The equilibrium at the nodes $j=1,2, \ldots, J$ of the truss in the directions $x_{1}$ (horizontally) and $x_{2}$ (vertically) is recorded as,

horizontally:

$$
\sum_{k=1}^{j-1}\left\{p_{2 e-1}+p_{2 e}\right\}\left(-r_{2 e-1}\right)+\sum_{k=j+1}^{J}\left\{p_{2 e-1}+p_{2 e}\right\} r_{2 e-1}=-b_{2 j-1}
$$

vertically:

$$
\sum_{k=1}^{j-1}\left\{p_{2 e-1}+p_{2 e}\right\}\left(-r_{2 e}\right)+\sum_{k=j+1}^{J}\left\{p_{2 e-1}+p_{2 e}\right\} r_{2 e}=-b_{2 j},
$$

where $b_{2 j-1}, b_{2 j}$ are components of external nodal forces at $j$ including possible reactions at supports. In eqns (9), the running index $e$ in the first sum on the left-hand side fulfils eqn (3), while $e$ in the second sum is defined in eqn (2). 


\section{LINEAR CASE}

In the linear case (without involving the buckling effect in the compressed members), eqn (8) is valid and linear equations of equilibrium (9) can be formed in a matrix form as,

$$
[R]\{P\}=\{B\},
$$

where the definition of $\{P\}$ is found in eqn (7), and

$$
\{B\}=-\left\{b_{1}, b_{2}, \ldots, b_{2 j-1}, b_{2 j}, \ldots, b_{2 J-1}, b_{2 J}\right\}^{\mathrm{T}} .
$$

The dimension of $[R]$ is $(2 J \times 2 N)$, the dimension of the vector $\{P\}$ is $(2 N \times 1)$, see $\operatorname{def}(7)$, and the dimension of the vector $\{B\}$ is $(2 J \times 1)$, see def (11). Till the end of this text the first forces in turn $\left\{p_{1}, \cdots, p_{N}\right\}$ are assumed tensile and $\left\{p_{N+1}, \cdots, p_{2 N}\right\}$ are taken compressed. The vector $\{C\}$ and the matrix $[R]$ also undergo the appropriate permutation.

The optimization problem may be formulated as: Find minimum $M(P)$ constrained by the condition:

$$
\{P\} \geq\{0\},
$$

normal stress at each member attains the admissible stress (see the definition of the vector $\{C\}$ in $\operatorname{def}(6))$.

Maximum number of members in a statically determined truss in $2 \mathrm{D}$ is

$$
K=2 J-3 .
$$

In the same time, the number $K$ is the rank of the linear system of eqns (10). In this sense, the optimization problem reduces to finding appropriate combination of $K$ members appropriately selected from all $N$ members, the forces in which obey the condition (12); all forces which respect the latter conditions create the basis.

Hereinafter, the above introduced relations can be split into sub-vectors and submatrices as,

$$
\{P\}=\left\{\left\{P_{\mathrm{I}}\right\},\left\{P_{\mathrm{E}}\right\}\right\}^{\mathrm{T}}, \quad\{C\}=\left\{\left\{C_{\mathrm{I}}\right\},\left\{C_{\mathrm{E}}\right\}\right\}^{\mathrm{T}}, \quad[R]=\left[\left[R_{\mathrm{I}}\right],\left[R_{\mathrm{E}}\right]\right],
$$

where the subscript I refers to the basis and subscript $\mathrm{E}$ is related to the zero off-basis forces, i.e.,

$$
\left\{P_{\mathrm{E}}\right\}=\{0\}
$$

Latter partitioning applies to eqns (8) and (10). Moreover, introduced matrix and vectors are further split into the linearly independent system 1 and the residual system 2 , which is on the system 1 dependent, 


$$
\left[R_{\mathrm{I}}\right]=\left[\begin{array}{c}
{\left[R_{\mathrm{I} 1}\right]} \\
{\left[R_{\mathrm{I} 2}\right]}
\end{array}\right], \quad\left[R_{\mathrm{E}}\right]=\left[\begin{array}{c}
{\left[R_{\mathrm{E} 1}\right]} \\
{\left[R_{\mathrm{E} 2}\right]}
\end{array}\right], \quad\{B\}=\left\{\begin{array}{c}
\left\{B_{1}\right\} \\
\left\{B_{2}\right\}
\end{array}\right\},
$$

where $\left[R_{\mathrm{I}}\right]$ has dimension $(K \times K)$, and the dimensions of the other matrices are obvious. Inasmuch eqn (15) is valid, it remains to solve $\left\{P_{\mathrm{I}}\right\}$ :

$$
\left\{P_{\mathrm{I}}\right\}=\left[R_{\mathrm{I} 1}\right]^{-1}\left\{B_{1}\right\}
$$

It is worth noting that applying Gaussian elimination to eqn (10), the identity $\left[R_{\mathrm{I} 1}\right]^{-1} \equiv[I]$ can be attained, where $[I]$ is the unit matrix, so that

$$
\left\{P_{\mathrm{I}}\right\}=\left\{B_{1}\right\}
$$

The expression of the overall mass $M$ is now reduced as, see eqn (8),

$$
M=\left\{C_{\mathrm{I}}\right\}^{\mathrm{T}} \cdot\left\{P_{\mathrm{I}}\right\}
$$

The matrices $\left[R_{\mathrm{I} 1}\right],\left[R_{\mathrm{E} 1}\right]$ can formally be recorded as,

$$
\left[R_{\mathrm{I} 1}\right]=\left[\left\{R_{1}\right\},\left\{R_{2}\right\}, \ldots,\left\{R_{K}\right\}\right], \quad\left[R_{\mathrm{E} 1}\right]=\left[\left\{R_{K+1}\right\},\left\{R_{K+2}\right\}, \ldots,\left\{R_{2 N}\right\}\right]
$$

where, obviously, $\left\{R_{i}\right\}, i=1,2, \ldots, K$, are column-vectors associated with the basis. Note that for simplicity $i=2 e-1$, as no buckling effect is assumed and the forces in the compressed members behave as that in tensile members.

Our goal is to enter a proper force from $\left\{P_{\mathrm{E}}\right\}$, which substitutes outgoing appropriately selected force from the current basis in order the overall mass of the truss to be reduced. In the same time, interchange of the respective column in $\left[R_{\mathrm{E} 1}\right]$ and the column in $\left[R_{\mathrm{I} 1}\right]$ has to be performed. Introducing an auxiliary matrix $[Z]$, each column $\left\{R_{K+1}\right\},\left\{R_{K+2}\right\}, \ldots,\left\{R_{2 N}\right\}$ in $\left[R_{\mathrm{E} 1}\right]$ can be formulated as a linear combination of the vectors in $\left[R_{\mathrm{I} 1}\right]$ :

$$
\left[R_{\mathrm{E} 1}\right]=\left[R_{\mathrm{I} 1}\right][Z],
$$

taking into consideration that there always exists such a basis for which $\left[R_{\mathrm{I}}\right]$ is regular. The auxiliary matrix, $[Z]$, has dimensions $(K \times(2 N-K))$. From eqn (21) immediately follows that

$$
[Z]=\left[R_{\mathrm{II}}\right]^{-1}\left[R_{\mathrm{E} 1}\right]=\left[\left\{Z_{K+1}\right\},\left\{Z_{K+2}\right\}, \ldots,\left\{Z_{2 N}\right\}\right] .
$$

If $\left[R_{\mathrm{I} 1}\right]$ is the unit matrix, $[Z]=\left[R_{\mathrm{E} 1}\right]$. 
Let us denote the new member $n$ which is selected as the potential member of the new basis (incoming member) and $m$ is the outgoing member. Eliminating the old system of constraints leads us to the following relations for the new configuration:

$$
R_{I 1 ; i j}^{\text {new }}=R_{I 1 ; i j}^{\text {old }}-\frac{Z_{\text {in }}^{\text {old }}}{Z_{m n}^{\text {old }}} R_{I 1 ; m j}^{\text {old }}, \text { or } R_{I 1 ; i j}^{\text {new }}=I_{i j}-\frac{Z_{\text {in }}^{\text {old }}}{Z_{m n}^{\text {old }}} R_{I 1 ; m j}^{\text {old }}
$$

and

$$
Z_{i j}^{\text {new }}=Z_{i j}^{\text {old }}-\frac{Z_{\text {in }}^{\text {old }}}{Z_{m n}^{\text {old }}} Z_{m j}^{\text {old }}, \quad b_{1 ; i}^{\text {new }}=b_{1 ; i}^{\text {old }}-\frac{Z_{i n}^{\text {old }}}{Z_{m n}^{\text {old }}} b_{1 ; m}^{\text {old }},
$$

where $i$ runs from 1 to $K$ but $i=m$, and $j=1, \cdots, 2 N$,

or

$$
R_{I 1 ; i j}^{\text {new }}=I_{i j}-Z_{i n}^{\text {old }} \bar{R}_{I 1 ; m j}^{\text {old }}, Z_{i j}^{\text {new }}=Z_{i j}^{\text {old }}-Z_{i n}^{\text {old }} \bar{Z}_{m j}^{\text {old }}, \quad b_{1 ; i}^{\text {new }}=b_{1 ; i}^{\text {old }}-Z_{i n}^{\text {old }} \bar{b}_{1 ; m}^{\text {old }}
$$

The barred quantities arise from dividing the introduced expression by $Z_{m n}^{\text {old }}$. After permutation of the columns $m$ and $\mathrm{n}$ including commutation of $\mathrm{cm}$ and $\mathrm{cn},\left[R_{I 1}{ }^{\text {new }}\right]$ turns to the unit matrix $I$ if

$R_{I 1, m j}^{\text {new }}=\frac{R_{I 1, m j}^{\text {old }}}{R_{I 1, m n}^{\text {old }}}$ and $b_{1 ; m}^{\text {new }}=\frac{b_{1 ; m}^{\text {old }}}{R_{I 1, m n}^{\text {old }}}$.

With respect to eqn (18), $b_{1 ; i}^{\text {old }}=p_{i}^{\text {old }}$, for $i=1, \cdots, K$ and eqn (25) becomes $p_{i}^{\text {new }}=p_{i}^{\text {old }}-Z_{\text {in }}^{\text {old }} \bar{p}_{i}^{\text {old }}, \bar{p}_{i}^{\text {old }}=\frac{p_{i}^{\text {old }}}{Z_{m n}^{\text {old }}}$, for $i \neq m$. If $i=m$ then $p_{m}^{\text {new }}=\frac{p_{m}^{\text {old }}}{Z_{m n}^{\text {old }}}=\bar{p}_{m}^{\text {old }}$. Recall that $p_{i}^{\text {old }}, \bar{p}_{i}^{\text {old }}$ and $Z_{\text {in }}^{\text {old }}$ are known from the previous step.

Let us denote $\bar{p}_{n}^{\text {old }}=\frac{p_{n}^{\text {old }}}{Z_{m n}^{\text {old }}}=\theta_{n}$, which is the true value of the force in the member $n$ entering the new configuration.

If one wants to introduce a new force $\theta_{n}>0$ in the member $n$, one obtains a new set of forces $P_{I}^{\text {new }}$ in the new basis:

$$
p_{i}^{\text {new }}=p_{i}^{\text {old }}-Z_{\text {in }}^{\text {old }} \theta_{n}
$$

The couple $(m, n)$ creates the key point in terms of the Simplex method, $m$ identifies the outgoing column and $n$ the ingoing one. In this way, the component of $\{C\}$ changes for $\{C\}^{n}$, in which the value of $c$ at the position $m$ interchanges with $c_{n}$ at the position $n$.

Then, the mass changes by the value, 


$$
\Delta M_{n}=\sum_{i=1}^{K} c_{i}^{\text {old }} p_{i}^{\text {old }}-\sum_{i=1}^{K} c_{i}^{\text {old }}\left(p_{i}^{\text {old }}-z_{i n}^{\text {old }} \theta_{n}\right)-c_{n}^{\text {old }} \theta_{n}=\sum_{i=1}^{K} c_{i}^{\text {old }} z_{i n}^{\text {old }} \theta_{n}-c_{n}^{\text {old }} \theta_{n}>0
$$

Note that the first term of the right-hand side in eqn (25) is the mass in the old configuration while the rest of the right-hand side is the mass of the new set up of members in the truss studied. As

$$
p_{i}^{\text {new }}=\left(p_{i}^{\text {old }}-Z_{i n}^{\text {old }} \theta_{n}\right) \rightarrow \min ,
$$

see eqn (26), optimality condition for selecting $n$ leads to:

$$
\theta_{n}=\min _{j \in(1, \ldots, K)}\left(\frac{P_{\mathrm{I}, j}}{Z_{n, j}}\right), \quad \text { successively for } n=K+1, \ldots, 2 N, \text { providing } \frac{P_{\mathrm{I}, j}}{Z_{n, j}}>0,
$$

so that the previous minimization leads to the identification of the member $m=j_{0} \in(1, \ldots, K)$ and fulfils the minimization criterion eqn (29). The member $m$ is leaving the basis, as for such a member the mass increases.

In the matrix form, eqn (27) provides

$$
\Delta M_{n}=\left(c_{n}^{\text {old }}-\left\{C_{I}^{\text {old }}\right\}^{\mathrm{T}}\left\{Z_{n}^{\text {old }}\right\}\right) \theta_{n}<0
$$

and as $\theta_{n}$ is required to be positive, the criterion for reduction of mass is,

$$
\Delta_{n}=\left(c_{n}^{\text {old }}-\left\{C_{I}^{\text {old }}\right\}^{\mathrm{T}}\left\{Z_{n}^{\text {old }}\right\}\right)<0 \text { for } n=K+1, \ldots, 2 N .
$$

The basis is then interchanged taking into account the member pertaining to the number $n$. In order for the mass to decrease in the current iteration, $\Delta_{n}$ in (26) should be minimized, i.e.,

$$
S=\min _{n=K+1, \ldots, 2 N} \Delta_{n} .
$$

The force $\theta_{n}$ reflects the interchange of the $n$-th force out of the current basis and the forces $\left\{P_{\mathrm{I}}\right\}$ to get improved $\left\{P_{\mathrm{I}}\right\}^{n}$, the component $P_{\mathrm{I}, c}$ in which is substituted by $\theta_{n}$, and which still fulfils the equilibrium at the nodes, i.e. $\left[R_{\mathrm{I} 1}\right]$ changes for $\left[R_{\mathrm{I} 1}\right]^{n}$ due to $\left[R_{\mathrm{E} 1, n}\right]$.

\section{BUCKLING}

In this section, a similar problem as that discussed in the previous section is studied, but is expanded for the assumption that the compressed members posses buckling property with slenderness ratio pertaining to the Euler area. The critical compressive stress $\sigma_{\text {crit }}$ in the member $e$ is equal to critical force $p_{2 e}$ divided by the cross-sectional area $A_{e}$.

Considering the safety margin $\eta_{\mathrm{E}}$, the Euler formula is expressed as, 


$$
\sigma_{\text {crit }}=\frac{p_{2 e}}{A_{e}}=\frac{\pi^{2} E_{e}}{\eta_{\mathrm{E}} \lambda_{e}^{2}}, \quad \lambda_{e}=\frac{l_{e}}{i_{e}}
$$

where $i_{e}=\sqrt{\frac{I_{e}}{A_{e}}}$ is the radius of gyration of the member cross-section with the area $A_{e}$, and $I_{e}$ is its moment of inertia.

Sometimes an additional coefficient is introduced to the formula (33), which expresses the number of waves, assuming that the member after buckling includes nodal points with zero deflections. Since the relation between the mass $m_{e}$ of the member $e$ and the force $p_{2 e}$ is sought, (33) can be rearranged as,

$$
m_{e}=\frac{\rho_{e} l_{e}^{2} A_{e}}{\pi \sqrt{\frac{E_{e} I_{e}}{\eta_{\mathrm{E}}}}} \sqrt{p_{2 e}} .
$$

Hence, for compressed member a new multiplier $c_{2 e}$ of the force $p_{2 e}$ is created as

$$
c_{2 e}=\frac{\rho_{e} l_{e}^{2} A_{e}}{\pi \sqrt{\frac{E_{e} I_{e}}{\eta_{\mathrm{E}}}}} .
$$

Introducing the expression for the mass of compressed members, eqn (19) is completed as,

$$
M=\left\{C^{+}\right\}^{\mathrm{T}}\left\{P_{+}\right\}+\left\{C^{-}\right\}^{\mathrm{T}}\left\{\sqrt{P_{-}}\right\}
$$

where $\left\{P_{+}\right\}=\left\{p_{1}, p_{3}, \ldots, p_{2 e-1}, \ldots, p_{2 K-1}\right\},\left\{P_{-}\right\}=\left\{p_{2}, p_{4}, \ldots, p_{2 e}, \ldots, p_{2 K}\right\}$. In what follows the index denotation is slightly changed. To each member index identification of internal forces is narrowed and only one integer is assigned either for tension or compression. Then,

$$
\begin{aligned}
& \left.\Delta M_{n}=\sum_{i=1}^{K} \underset{\substack{c_{i}^{+} p_{i} \\
\text { tension }}}{\left.c_{i}^{-} \sqrt{p_{i}}\right]}\right]-\sum_{\substack{\text { compression } \\
\text { tel }}}^{K}\left[c_{i}^{+}\left(p_{i}-z_{\text {in }} \theta_{n}\right)+c_{i}^{-} \sqrt{p_{i}-z_{i n} \theta_{n}}\right]- \\
& \underset{\text { tension }}{c_{n}^{+} p_{n}}+\underset{\text { compression }}{c_{n}^{-} \sqrt{p_{n}}}>0 .
\end{aligned}
$$

The next approach is the same as that introduced in the previous section.

\section{EXTERNAL STATIC INDETERMINACY}

So far, the structure was considered as externally determinate. If it is not so, the structure possesses too many external constraints, the deformation conditions have to be employed. At the positions the redundant forces are located, unit displacement impulses in the direction of acting reactions are applied to get 


$$
p_{i}=p_{o i}+\sum_{j=1}^{S} \Theta_{i j} X_{j}
$$

where $p_{i}$ is the improved force due to external indeterminacy in the member $i, p_{o i}$ is the force in the same member $i$ from the loading the basic statically determined structure, $S$ is the number of external constraints -3 in $2 \mathrm{D}, \Theta_{i j}$ is the axial force in the member $i$ due to the unit load at the superfluous support $j, X_{j}$ is the real value of the reaction. This will be detected from the deformation condition in the sense of force method.

\section{EXAMPLES}

Suppose the protective wall depicted in Fig. 1. Various combinations of geometry and number of trusses in structural systems are studied. In case the buckling takes place, the constant in eqns (34), (35) is selected as: $\frac{1}{A_{e}} \sqrt{\frac{I_{e}}{\eta_{\mathrm{E}}}}=0.33$. The optimal truss structures are observed in certain typical scenarios. In Fig. 2, the simplest two span steel truss is investigated, loaded along the lower chord. In the results of iteration shown, two members are obviously equal to zero while in the optimal set up even four members are annihilated, although the number of compressed members increases.

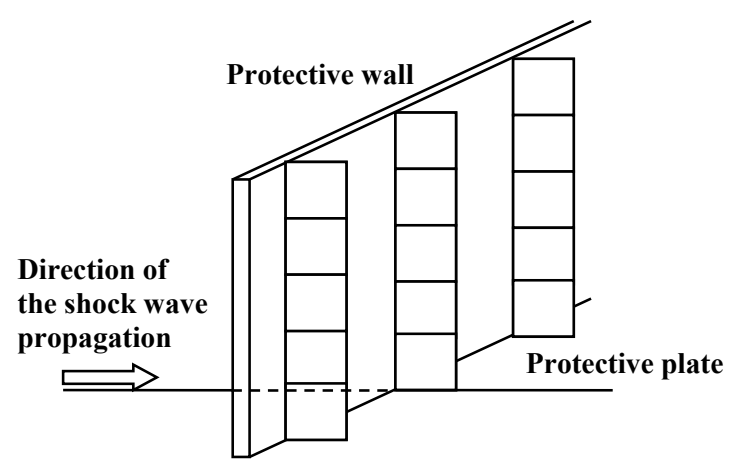

Figure 1: Protective wall with the protective plate and system of supporting trusses.
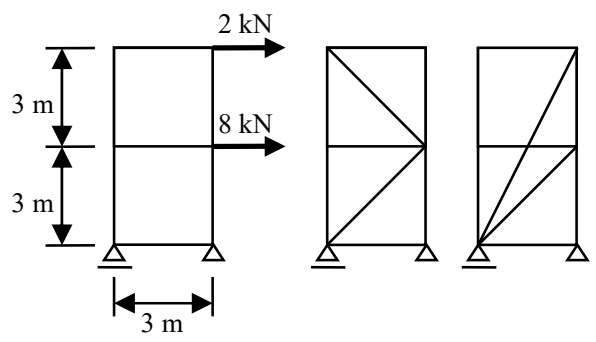

Figure 2: Two span truss optimized, iteration $(M=378 \mathrm{~kg} \approx 833 \mathrm{lbs})$, and optimal shape. $(M=291 \mathrm{~kg} \approx 642 \mathrm{lbs})$. 
In the next example, the optimum possesses almost the same configuration as that attained in previous iteration. The load distribution corresponds to the source of the explosion that is placed on the ground.

In Fig. 4, again a three-span truss is studied with the loading distributed along the lower chord, which simulates the influence of the shock wave leaving the structure and causes sucking. Comparing the geometry of the optimized truss structures displayed in Fig. 3 and Fig. 4, the difference appears only in the field most distant from the supports. The value of the optimal mass is again not significant in comparison with the previous iteration step.

With respect to a relatively small difference of mass, the structure displayed in Fig. 4 can be considered by mass equivalent. Thus, the decisive factor consists in an architectural and construction view, which of the two alternatives can be evaluated as appropriate. In many cases the optimized structures are of the shapes, which are for architects unacceptable. Then such an optimized structure may be considered as a design draft and serve a support for final design decision.
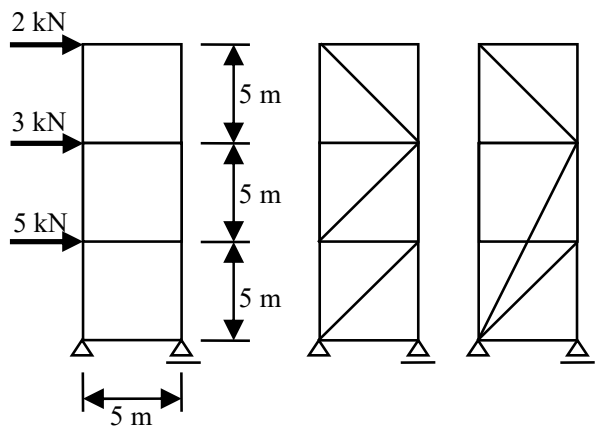

Figure 3: Three span truss in optimization, upper chord loaded, iteration ( $M=745 \mathrm{~kg} \approx 1642 \mathrm{lbs})$ and optimal shape $(M=741 \mathrm{~kg} \approx 1634 \mathrm{lbs})$.
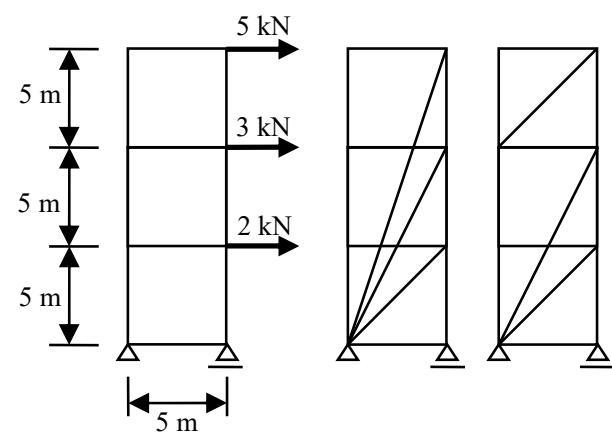

Figure 4: Three span truss is optimized, lower chord loaded, iteration $(M=560 \mathrm{~kg} \approx 1235 \mathrm{lbs})$ and optimal shape $(M=542 \mathrm{~kg} \approx 1195 \mathrm{lbs})$. 

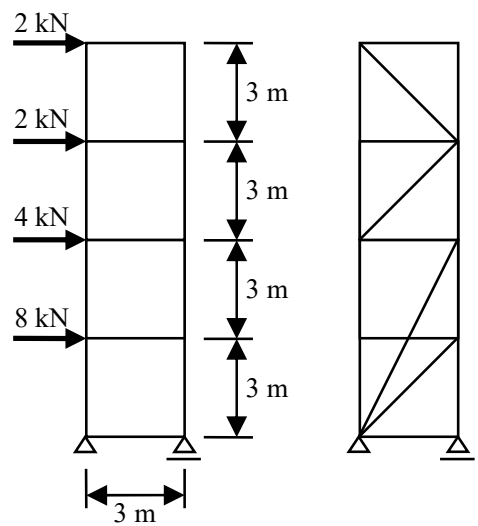

Figure 5: Four span truss in optimization, iteration $(M=922 \mathrm{~kg} \approx 2033 \mathrm{lbs})$ and optimal shape $(M=685 \mathrm{~kg} \approx 1510 \mathrm{lbs})$.

The last case involves four span truss structure loaded by the shock wave positioned again in the terrain. Here the minimum mass is attained again for some members with zero forces; two members are annihilated, for example. Optimum is significantly different from the previous iteration.

\section{CONCLUSION}

Nowadays, the protection against explosions belongs to the main issue in civil engineering. In this paper, optimal shapes of truss structures serving as supports of protective wall are sought. The solution starts with the basic ideas of classical simplex method, which are partly implemented to the approach of this optimization. A very important factor is the inclusion of buckling of compressed members in the formulation of the problem.

In the case of a more complex structure, the compressed members are firstly changed to the tensile ones till the internal equilibrium forces the tensile members to be partly replaced by compressed. The latest iteration steps apply only to the tension members; the effect of compressed members to the mass remains always constant. With respect to a relatively small difference of mass, the structure displayed in Fig. 4 can be considered almost similar. This can happen often; such a phenomenon is a positive result, as the decisive factor consists in an architectural and construction opinion, which of the alternatives can be evaluated as more appropriate. Solved examples deal with externally determinate structures only, but it is easy to optimize the design using the proposed method also in the case of externally indeterminate trusses, as is apparent.

\section{ACKNOWLEDGEMENT}

Financial support by the Grant agency of the Czech Republic No. 17-04204S is greatly acknowledged.

\section{REFERENCES}

[1] Fleron, P., The minimum weight layout of trusses. Bygnings statiske meddelelser 35(3), 1964. 
[2] Giovino, G., Opati, P., Garbati, S. \& Bontempi, F., Blast resistance assessment of concrete wall panels: experimental and numerical investigations. International Journal of Protective Structures, 5(3), pp. 349-366, 2014.

[3] Procházka, P.P., Deterioration of FRC plate due to explosion and change of temperature. Key Engineering Materials, pp. 525-526, pp. 185-188, 2013.

[4] Lavarnway, D. \& Pollino, M., Mitigation of air-blast pressure impulses on building envelopes through blast resistant ductile connectors. Journal of Engineering and Architecture, 3(2), pp. 9-24, 2015.

[5] Prochazka, P.P., Kravtsov, A.N. \& Lok T.S., Assessment of laminated cylindrical arch loaded by a shock wave. International Journal of Protective Structures, 2(2), pp. 267-282, 2011.

[6] Prochazka, P.P., Effect of explosion and fire on underground structures. International Journal of Protective Structures, 4(4), pp. pp. 505-520, 2014.

[7] Prochazka, P.P. \& Lok, T.-S., Explosion and temperature resistance of underground structures by free hexagons. Key Engineering Materials, 488-489, pp. 678-681, 2012.

[8] Smith, P.D., Blast walls for structural protection against high explosive threats: a review. International Journal of Protective Structures, 1(1), pp. 67-84, 2010.

[9] Kaur, G., Bansal, R.S. \& Kumar, S., Shape Optimization of Roof Truss. International Journal of Engineering Research \& Technology, 5(6), pp. 696-700, 2016.

[10] Camp, C.V. \& Farshchin, M., Design of space trusses using modified teachinglearning based optimization. Engineering Structures, 62-63, pp. 87-97, 2014.

[11] Kaveh, A., Sheikholeslami, R., Talatahari, S. \& Keshvari-Ilkhichi, M., Chaotic swarming of particles: A new method for size optimization of truss structures. Advances in Engineering Software, 67, pp. 136-147, 2014.

[12] Felkner, J., Chatzi, E. \& Kotnik, T., Interactive truss design using Particle Swarm Optimization and NURBS curves. Journal of Building Engineering, 4, pp. 60-74, 2015.

[13] Hasançebi, O. \& Erbatur, F., Layout optimisation of trusses using simulated annealing. Advances in Engineering Software, 33(7)-(10), pp. 681-696, 2002.

[14] Frans, R. \& Arfiadi, Y., Sizing, shape, and topology optimizations of roof trusses using hybrid genetic algorithms, Procedia Engineering 95, pp. 185-195, 2014. 